\title{
EXISTENCE OF WEAK SOLUTIONS FOR A CLASS OF SEMILINEAR STOCHASTIC WAVE EQUATIONS
}

\author{
CARLO MARINELLI AND LLUÍS QUER-SARDANYONS
}

\begin{abstract}
We prove existence of weak solutions (in the probabilistic sense) for a general class of stochastic semilinear wave equations on bounded domains of $\mathbb{R}^{d}$ driven by a possibly discontinuous square integrable martingale.
\end{abstract}

\section{INTRODUCTION}

The purpose of this paper is to prove existence of weak solutions, in the probabilistic sense, for a class of semilinear stochastic wave equations of the type

$$
\frac{\partial^{2} u}{\partial t^{2}}(t, x)-\Delta u(t, x)+\beta(u(t, x))=\dot{\eta}(t, x)
$$

on a bounded space-time domain $[0, T] \times D \subset \mathbb{R}^{1+d}$, complemented with suitable boundary conditions. Here $\beta$ denotes a maximal monotone graph in $\mathbb{R} \times \mathbb{R}$ and $\dot{\eta}$ stands for the time derivative of a stochastic integral with respect to a Hilbert space-valued (possibly discontinuous) martingale which may depend on $u$. Of course (11) is only a formal expression, whose corresponding rigorous stochastic evolution equation, as well as the definition of solution, will be given below.

Existence of solutions for stochastic PDEs such as (11) cannot be obtained (to the best of our knowledge) simply as an application of some general technique. In particular, on the one hand (11) cannot be cast in the variational setting of Pardoux [31] and Krylov-Rozovskii [19], as the equation is not of monotone type, in spite of $\beta$ being monotone. On the other hand, since the nonlinear term $\beta$ does not satisfty any (even local) Lipschitz condition, the semigroup approach does not seem to be applicable directly either. However, major efforts have been devoted, especially in recent years, to obtain existence, uniqueness, and regularity results for classes of stochastic PDEs that do not fall into any standard framework (see e.g. 2]), and our work is a contribution in this direction. In the particular case of stochastic wave equations of the form (11), some well-posedness results in the mild sense have been obtained assuming that $\beta$ has sufficiently slow polynomial growth and that $\eta$ is a stochastic integral with respect to a

Date: 21 October 2011.

2000 Mathematics Subject Classification. 60H15; 60G57.

Key words and phrases. Stochastic PDEs, nonlinear wave equations, monotone operators, infinite dimensional semimartingales.

Part of the work for this paper was carried out while the first author was visiting the Department of Statistics of Purdue University supported by a MOIF fellowship, and while the second author was visiting the CIRM at the University of Trento supported by a fellowship of the Fondazione Bruno Kessler. The second author was also supported by the grant MEC-FEDER Ref. MTM2009-08869 of the Ministerio de Ciencia e Innovación, Spain. We thank Viorel Barbu for helpful conversations on the topic of this paper. 
Wiener process - see e.g. [7, 27, 29]. Moreover, Ondreját [30] has recently proved that for any $\beta$ continuous and polynomially growing, and $\eta$ a stochastic integral with respect to a spatially homogeneous Wiener process with finite spectral measure, (1) admits a (suitably defined) global weak solution. More precisely, he proves the existence of a solution to an infinite dimensional martingale problem associated to (1).

Let us also recall that there exist in the literature several well-posedness results for stochastic wave equations with Lipschitz continuous drift, among which [16, 33, 34, 35, where the semigroup approach is used, and [6, 8, 9, 10, 11, 28, that adopt the random field approach (à la Walsh [41]).

Our approach, whose origins can be traced back to [40, \$1.6] (cf. also [18, Section 2.6] and [32]), is entirely different and relies instead on Skorohod's representation theorem and weak convergence results for stochastic integrals with respect to general martingales. This route allows us to avoid going through the martingale problem for an equation with discontinuous noise, which is already quite involved in finite dimensions (see e.g. [13, 23]). Roughly speaking, our proof proceeds as follows: we obtain a priori estimates for solutions of regularized equations (i.e. with smoother $\beta$ ) which imply tightness in an appropriate topology. We can thus construct a sequence of processes converging almost surely on a different probability space. The final step consists in showing that the limit process is a weak solution of the equation of interest, in a sense made precise below. A key ingredient in this step is played by a convergence result in 4] (see Theorem 10 below). Let us also mention that unfortunately we cannot prove well-posedness, but only existence of a solution. Nonetheless, this is consistent with the deterministic case (i.e. with $\eta \equiv 0$ ), for which, under the present generality of $\beta$, no well-posedness results are known, even though existence of global weak solutions is known in some cases (see e.g. [4, 39]).

The paper is organized as follows: in Section 2 we introduce notation, recall some basic preliminaries needed throughout the paper, define the concept of solution to (1), and state the main result of the paper (Theorem 3 ). Section 3 contains a few auxiliary results on mild solutions of stochastic evolution equations with Lipschitz nonlinearities that are needed in the proof of the main result and that might be of independent interest. Finally, Section 4 is devoted to the proof of the main result.

\section{MAIN RESUlT}

2.1. Notation and preliminaries. Let $D \subset \mathbb{R}^{d}$ be a bounded domain with smooth boundary $\partial D$ and $T$ a fixed positive real number. We shall use standard notation for spaces of integrable functions and Sobolev spaces on $D$. In particular, $H_{0}^{1}(D)$ will denote the closure of $C_{c}^{\infty}(D)$ in the topology of $H^{1}(D)$. When no confusion may arise, we shall suppress the indication of the domain $D$, so that $L^{2}$ and $H_{0}^{1}$ stand for $L^{2}(D)$ and $H_{0}^{1}(D)$, respectively. We shall denote the Laplace operator on $D$ with Dirichlet boundary conditions by $\Delta$.

Let $\beta$ be a maximal monotone graph in $\mathbb{R} \times \mathbb{R}$ such that $\operatorname{dom}(\beta)=\mathbb{R}$ and $0 \in \beta(0)$. Recall that a graph $\beta$ is called monotone if, for any $x_{1}, x_{2} \in \mathbb{R}, b_{1} \in \beta\left(x_{1}\right), b_{2} \in \beta\left(x_{2}\right)$, one has $\left(b_{1}-b_{2}\right)\left(x_{1}-x_{2}\right) \geq 0$, and a monotone graph $\beta$ is maximal if it is not properly contained in any other monotone graph. Let us recall that, if $f_{0}: \mathbb{R} \rightarrow \mathbb{R}$ is an increasing function, the graph $f: x \mapsto\left[f_{0}(x-), f_{0}(x+)\right] \cap \mathbb{R}$ is a monotone graph in $\mathbb{R} \times \mathbb{R}$. As a 
matter of fact, all maximal monotone graphs in $\mathbb{R} \times \mathbb{R}$ are constructed in this manner (cf. [5, Exemple 2.3.1]). We shall also assume, without loss of generality (see e.g. [1, p. 71]), that $\beta=\partial j$ for a continuous convex function $j: \mathbb{R} \rightarrow \mathbb{R}, j(x) \geq 0$ for all $x \in \mathbb{R}$. Here $\partial$ stands for the subdifferential in the sense of convex analysis, i.e.

$$
\partial j(x):=\{y \in \mathbb{R}: j(z)-j(x) \geq y(z-x) \quad \forall z \in \mathbb{R}\} .
$$

If $j$ is differentiable at $x$, then $\partial j(x)$ reduces to a single point and coincides with $f^{\prime}(x)$. For all notions of convex analysis and the theory of monotone operators used in the paper we refer to [1, 5].

For any real Hilbert spaces $E$ and $F$, let us denote the space of linear bounded operators from $E$ to $F$ by $\mathcal{L}(E \rightarrow F)$, and its subspace of Hilbert-Schmidt operators from $E$ to $F$ by $\mathcal{L}_{2}(E \rightarrow F)$. Given a symmetric nonnegative nuclear operator $R \in \mathcal{L}(E \rightarrow E)$, we shall use the notation $\mathcal{L}_{2}^{R}(E \rightarrow F):=\mathcal{L}_{2}\left(R^{1 / 2} E \rightarrow F\right)$, and $|B|_{R}:=\left|B R^{1 / 2}\right|_{\mathcal{L}_{2}(E \rightarrow F)}$.

Given a (fixed) real separable Hilbert space $\mathcal{H}$, let $M$ be a $\mathcal{H}$-valued square integrable martingale defined on a complete filtered probability space $\left(\Omega, \mathcal{F},\left\{\mathcal{F}_{t}\right\}_{t \in[0, T]}, \mathbb{P}\right)$, satisfying the "usual" conditions. Let $Q_{M}$ denote the martingale covariance of $M$, i.e. the (unique) predictable process with values in $\mathcal{L}_{1}^{+}(\mathcal{H})$, the space of symmetric nonnegative nuclear operators on $\mathcal{H}$, such that $\langle\langle M\rangle\rangle(t)=\int_{0}^{t} Q_{M}(s) d\langle M\rangle(s)$, where $\langle M\rangle$ and $\langle\langle M\rangle\rangle$ stand for the Meyer process and the operator angle bracket of $M$, respectively. Here and in the following we use standard notation and terminology for infinite dimensional stochastic calculus, for which we refer to [25] (see also [36]). Unless otherwise stated, we shall always assume that there exists $Q \in \mathcal{L}_{1}^{+}(\mathcal{H})$ such that

$$
\langle\langle M\rangle\rangle(t)-\langle\langle M\rangle\rangle(s) \leq(t-s) Q
$$

for all $0 \leq s \leq t \leq T$. Recall that, if $X$ is a further Hilbert space, then any predictable process $\Phi:[0, T] \rightarrow \mathcal{L}_{2}^{Q}(\mathcal{H} \rightarrow X)$ is integrable with respect to $M$, and it holds

$$
\mathbb{E}\left|\int_{0}^{T} \Phi(s) d M(s)\right|_{X}^{2}=\mathbb{E} \int_{0}^{T}|\Phi(s)|_{Q_{M}}^{2} d\langle M\rangle(s) \leq \mathbb{E} \int_{0}^{t}|\Phi(s)|_{Q}^{2} d s .
$$

Note that any martingale Lévy process (in particular, a Wiener process) with nuclear covariance operator satisfies the hypotheses imposed on $M$.

The space of $E$-valued random variables with finite $p$-th moment will be denoted by $\mathbb{L}^{p}(E)$, without explicit mention of the underlying probability space if no confusion may arise. Finally, the set of càdlàg functions defined on $[0, T]$ and taking values in $E$ (here $E$ can be any Polish space) will be denoted by $D([0, T] \rightarrow E)$.

We shall write $a \lesssim b$ to mean that there exists a constant $N>0$ such that $a \leq N b$.

2.2. Main result. Let us begin specifying the definition of solution for (1), which must be interpreted as the system of equations

$$
\left\{\begin{array}{l}
d u(t)=v(t) d t \\
d v(t)-\Delta u(t) d t+\beta(u(t)) d t=G_{0}(u(t-)) d M(t)
\end{array}\right.
$$

with initial conditions $(u(0), v(0))=\left(u_{0}, v_{0}\right) \in \mathbb{L}^{2}\left(H_{0}^{1} \times L^{2}\right)$, so that, on a formal level,

$$
\eta(t, \cdot)=\int_{0}^{t} G_{0}(u(s-)) d M(s) .
$$


We shall assume throughout the paper that $G_{0}: L^{2} \rightarrow \mathcal{L}_{2}^{Q}\left(\mathcal{H} \rightarrow L^{2}\right)$ is Lipschitz continuous.

Definition 1. A weak solution of equation (3) consists of a stochastic basis $\overline{\mathcal{B}}:=$ $(\bar{\Omega}, \overline{\mathcal{F}}, \overline{\mathbf{F}}, \overline{\mathbb{P}}), \overline{\mathbf{F}}:=\left\{\overline{\mathcal{F}}_{t}\right\}_{t \leq T}$, an $\mathcal{H}$-valued $\overline{\mathbf{F}}$-martingale $\bar{M}$, and an $\overline{\mathbf{F}}$-adapted càdlàg process $\bar{u}:[0, T] \rightarrow L^{2}$, both defined on $\overline{\mathcal{B}}$, such that the following conditions are satisfied:

(i) $\bar{M}$ has the same law of $M$ in $D\left([0, T] \rightarrow L^{2}\right)$;

(ii) there exists $g \in L^{1}(\bar{\Omega} \times[0, T] \times D)$ such that $g(\bar{\omega}, t, x) \in \beta(\bar{u}(\bar{\omega}, t, x))$ for $\overline{\mathbb{P}} \otimes$ Leba.a. $(\bar{\omega}, t, x)$ and

$$
\begin{array}{r}
\bar{u}(t)+\int_{0}^{t} \frac{1}{\sqrt{-\Delta}} \sin ((t-s) \sqrt{-\Delta}) g(s) d s=\cos (t \sqrt{-\Delta}) u_{0}+\frac{1}{\sqrt{-\Delta}} \sin (t \sqrt{-\Delta}) v_{0} \\
+\int_{0}^{t} \frac{1}{\sqrt{-\Delta}} \sin ((t-s) \sqrt{-\Delta}) G_{0}(\bar{u}(s-)) d \bar{M}(s)
\end{array}
$$

$\overline{\mathbb{P}}$-a.s. for all $t \leq T$.

Remark 2. Equation (44) is motivated by the classical Duhamel's representation of solutions to the linear non-homogeneous wave equation (see e.g. [39, §4.1]). For the right functional spaces in which the integrals have to be understood we refer to the proof of Theorem 3 below.

Our main result is the following theorem, which establishes existence of a weak solution for (3).

Theorem 3. Assume that $u_{0} \in \mathbb{L}^{2}\left(H_{0}^{1}\right), v_{0} \in \mathbb{L}^{2}\left(L^{2}\right)$ and $\mathbb{E}\left|j\left(u_{0}\right)\right|_{L^{1}}<\infty$. Then equation (3) admits a weak solution.

\section{AuXiliary RESUlts}

We collect in this section some auxiliary results on regularization and a priori estimates for mild solutions of stochastic evolution equations (with Lipschitz nonlinearities) which will be used in the next section. These results are not tied in any ways to the specific wave equation introduced above.

In addition to the notation already introduced, throughout this section we shall fix a real separable Hilbert space $X$ and a linear maximal monotone operator $A: \operatorname{dom}(A) \subset$ $X \rightarrow X$, and we shall set, for the sake of compactness of notation, $\mathcal{L}_{2}^{Q}:=\mathcal{L}_{2}^{Q}(\mathcal{H} \rightarrow X)$. The norm and the scalar product in $X$ will be denoted by $|\cdot|$ and $\langle\cdot, \cdot\rangle$, respectively. There will be no risk of confusion with the notation used in the other sections.

The following simple result can be proved essentially as [37, Lemma 2.4.1], thus we omit its proof (cf. also [36, p. 114]).

Lemma 4. Let $\Phi:[0, T] \rightarrow \mathcal{L}_{2}^{Q}$ be a predictable process such that

$$
\mathbb{E} \int_{0}^{T}|\Phi(s)|_{Q}^{2} d s<\infty
$$


and $B: X \rightarrow X$ a bounded linear operator. Then

$$
B \int_{0}^{t} \Phi(s) d M(s)=\int_{0}^{t} B \Phi(s) d M(s)
$$

$\mathbb{P}$-a.s. for all $t \in[0, T]$.

As an immediate consequence we also have the following corollary, which exploits the well-known fact that $-A$ and its associated semigroup and resolvent commute.

Corollary 5. Let $\Phi$ be as in Lemma四 and set $\Phi^{(\varepsilon)}:=(I+\varepsilon A)^{-1} \Phi, \varepsilon>0$. Then

$$
A \int_{0}^{t} e^{-(t-s) A} \Phi^{(\varepsilon)}(s) d M(s)=\int_{0}^{t} A e^{-(t-s) A} \Phi^{(\varepsilon)}(s) d M(s)
$$

$\mathbb{P}$-a.s. for all $t \in[0, T]$.

In order to obtain a priori estimates for mild solutions we shall approximate by strong solutions. The next lemma is a tool for the proposition to follow. Since we have not been able to find the proofs of these results in the literature, we will include them for the reader's convenience and the sake of completeness.

Lemma 6. For $\varepsilon>0$, let $Y^{(\varepsilon)}$ be the unique mild solution of the equation

$$
d Y^{(\varepsilon)}(t)+A Y^{(\varepsilon)}(t) d t+f(t) d t=\Phi^{(\varepsilon)}(t) d M(t), \quad Y^{(\varepsilon)}(0)=Y_{0},
$$

where $Y_{0} \in \operatorname{dom}(A), f \in L^{1}([0, T] \rightarrow \operatorname{dom}(A)) \mathbb{P}$-a.s., and $\Phi, \Phi^{(\varepsilon)}$ are as in Corollary 5 . Then $Y^{(\varepsilon)}$ is a strong solution of (7).

Proof. As a first step, we shall assume that $f$ and $Y_{0}$ are identically zero, so that

$$
\begin{aligned}
Y^{(\varepsilon)}(t) & =\int_{0}^{t} e^{-(t-s) A} \Phi^{(\varepsilon)}(s) d M(s) \\
& =(I+\varepsilon A)^{-1} \int_{0}^{t} e^{-(t-s) A} \Phi(s) d M(s) \quad \forall t \in[0, T] .
\end{aligned}
$$

We want to prove that one has

$$
Y^{(\varepsilon)}(t)=-\int_{0}^{t} A Y^{(\varepsilon)}(s) d s+\int_{0}^{t} \Phi^{(\varepsilon)}(s) d M(s) \quad \forall t \in[0, T] .
$$

On the one hand, it is clear that the stochastic integral on the right-hand side of (9) is well-defined. On the other hand, since $Y^{(\varepsilon)}(s) \in \operatorname{dom}(A) \mathbb{P}$-a.s. for all $s \in[0, t]$, applying 
a stochastic Fubini's theorem (e.g. as formulated in [22]) and Corollary [5, we have

$$
\begin{aligned}
\int_{0}^{t} A Y^{(\varepsilon)}(s) d s & =\int_{0}^{t}\left(A \int_{0}^{s} e^{-(s-r) A} \Phi^{(\varepsilon)}(r) d M(r)\right) d s \\
& =\int_{0}^{t}\left(\int_{0}^{s} A e^{-(s-r) A} \Phi^{(\varepsilon)}(r) d M(r)\right) d s \\
& =\int_{0}^{t}\left(\int_{r}^{t} A e^{-(s-r) A} \Phi^{(\varepsilon)}(r) d s\right) d M(r) \\
& =\int_{0}^{t}\left(-e^{-(t-r) A} \Phi^{(\varepsilon)}(r)+\Phi^{(\varepsilon)}(r)\right) d M(r) \\
& =-\int_{0}^{t} e^{-(t-r) A} \Phi^{(\varepsilon)}(r) d M(r)+\int_{0}^{t} \Phi^{(\varepsilon)}(r) d M(r),
\end{aligned}
$$

which is equivalent to (9), in view of (8).

In the general case (i.e. without assuming that $f$ and $Y_{0}$ are identically zero), the mild solution of (7) is given by

$$
Y^{(\varepsilon)}(t)+\int_{0}^{t} e^{-(t-s) A} f(s) d s=e^{-t A} Y_{0}+\int_{0}^{t} e^{-(t-s) A} \Phi^{(\varepsilon)}(s) d M(s)
$$

for all $t \in[0, T]$. Setting

$$
Z^{(\varepsilon)}(t):=Y^{(\varepsilon)}(t)+\int_{0}^{t} e^{-(t-s) A} f(s) d s-e^{-t A} Y_{0}
$$

we have $Z^{(\varepsilon)}(t) \in \operatorname{dom}(A)$ for all $t \in[0, T]$ and

$$
Z^{(\varepsilon)}(t)=\int_{0}^{t} e^{-(t-s) A} \Phi^{(\varepsilon)}(s) d M(s)
$$

for all $t \in[0, T]$. Therefore, by the first part of the proof, $Z^{(\varepsilon)}$ also verifies

$$
Z^{(\varepsilon)}(t)=-\int_{0}^{t} A Z^{(\varepsilon)}(s) d s+\int_{0}^{t} \Phi^{(\varepsilon)}(r) d M(r) \quad \forall t \in[0, T],
$$

which implies, recalling (10) and applying Fubini's theorem,

$$
Y^{(\varepsilon)}(t)+\int_{0}^{t} A Y^{(\varepsilon)}(s) d s+\int_{0}^{t} f(s) d s=Y_{0}+\int_{0}^{t} \Phi^{(\varepsilon)}(r) d M(r),
$$

thus concluding the proof.

The next proposition establishes a priori estimates for the mild solution of an equation with Lipschitz nonlinearities.

Proposition 7. Let $y$ be the mild solution of

$$
d y(t)+A y(t) d t=f(y(t)) d t+\Phi_{0}(y(t-)) d M(t), \quad y(0)=y_{0},
$$

where $f: X \rightarrow X$ and $\Phi_{0}: X \rightarrow \mathcal{L}_{2}^{Q}$ are Lipschitz continuous. Then

$$
\mathbb{E} \sup _{t \leq T}|y(t)|^{2} \lesssim 1+\mathbb{E}\left|y_{0}\right|^{2}+\mathbb{E} \sup _{t \leq T} \int_{0}^{t}\langle f(y(s)), y(s)\rangle d s+\int_{0}^{T}\left(\mathbb{E} \sup _{r \leq s}|y(r)|^{2}\right) d s .
$$


Proof. Let us recall that the mild solution $y$ is unique, càdlàg, and satisfies (see e.g. [17])

$$
\mathbb{E} \sup _{t \leq T}|y(t)|^{2}<+\infty
$$

Set $b(\cdot):=f(y(\cdot)), \Phi(\cdot):=\Phi_{0}(y(\cdot-))$. Then $y$ is the mild solution of

$$
d y(t)+A y(t) d t=b(t) d t+\Phi(t) d M(t), \quad y(0)=y_{0},
$$

that is

$$
y(t)=e^{-t A} y_{0}+\int_{0}^{t} e^{-(t-s) A} b(s) d s+\int_{0}^{t} e^{-(t-s) A} \Phi(s) d M(s)
$$

$\mathbb{P}$-a.s. for all $t \in[0, T]$. Applying $(I+\varepsilon A)^{-1}$ to both sides we have, in view of Lemma 4 ,

$$
y^{(\varepsilon)}(t)=e^{-t A} y_{0}^{(\varepsilon)}+\int_{0}^{t} e^{-(t-s) A} b^{(\varepsilon)}(s) d s+\int_{0}^{t} e^{-(t-s) A} \Phi^{(\varepsilon)}(s) d M(s)
$$

where we have used the notation $h^{(\varepsilon)}:=(I+\varepsilon A)^{-1} h$, for any $h$ for which it makes sense. In other words, $y^{(\varepsilon)}$ is the mild solution of

$$
d y^{(\varepsilon)}(t)+A y^{(\varepsilon)}(t) d t=b^{(\varepsilon)}(t) d t+\Phi^{(\varepsilon)}(t) d M(t), \quad y^{(\varepsilon)}(0)=y_{0}^{(\varepsilon)} .
$$

On the other hand, notice that we can apply Lemma 6 since, by (2) and the Lipschitz continuity of $\Phi_{0}$,

$$
\mathbb{E} \int_{0}^{T}\left|\Phi_{0}(y(s))\right|_{Q}^{2} d s \lesssim 1+\mathbb{E} \sup _{t \leq T}|y(t)|^{2}<+\infty .
$$

Therefore, $y^{(\varepsilon)}$ is also a strong solution of the previous equation, for which we can apply Itô's formula for the square of the norm, obtaining

$$
\begin{aligned}
\left|y^{(\varepsilon)}(t)\right|^{2}= & \left|y_{0}^{(\varepsilon)}\right|^{2}+2 \int_{0}^{t}\left\langle y^{(\varepsilon)}(s-), d y^{(\varepsilon)}(s)\right\rangle+\left[y^{(\varepsilon)}\right](t) \\
= & \left|y_{0}^{(\varepsilon)}\right|^{2}-2 \int_{0}^{t}\left\langle A y^{(\varepsilon)}(s), y^{(\varepsilon)}(s)\right\rangle d s+2 \int_{0}^{t}\left\langle b^{(\varepsilon)}(s), y^{(\varepsilon)}(s)\right\rangle d s \\
& +2 \int_{0}^{t}\left\langle y^{(\varepsilon)}(s-), \Phi^{(\varepsilon)}(s) d M(s)\right\rangle+\left[\Phi^{(\varepsilon)} \cdot M\right](t) \\
\leq & \left|y_{0}\right|^{2}+2 \int_{0}^{t}\left\langle b^{(\varepsilon)}(s), y^{(\varepsilon)}(s)\right\rangle d s+2 \int_{0}^{t}\left\langle y^{(\varepsilon)}(s-), \Phi^{(\varepsilon)}(s) d M(s)\right\rangle+[\Phi \cdot M](t),
\end{aligned}
$$

where the inequality follows by the monotonicity of $A$ and the contractivity of $(I+\varepsilon A)^{-1}$. Thus we also have

$$
\begin{aligned}
\mathbb{E} \sup _{t \leq T}\left|y^{(\varepsilon)}(t)\right|^{2} \leq \mathbb{E} \mid & \left.y_{0}\right|^{2}+2 \mathbb{E} \sup _{t \leq T} \int_{0}^{t}\left\langle b^{(\varepsilon)}(s), y^{(\varepsilon)}(s)\right\rangle d s \\
& +2 \mathbb{E} \sup _{t \leq T} \int_{0}^{t}\left\langle y^{(\varepsilon)}(s-), \Phi^{(\varepsilon)}(s) d M(s)\right\rangle+\mathbb{E}[\Phi \cdot M](T) .
\end{aligned}
$$

We are going to get a uniform (with respect to $\varepsilon$ ) estimate for the third term on the right-hand side. For this, consider the local martingale

$$
N_{\varepsilon}(t):=\int_{0}^{t}\left\langle y^{(\varepsilon)}(s-), \Phi^{(\varepsilon)}(s) d M(s)\right\rangle, \quad t \in[0, T]
$$


for which Davis' and Young's inequalities yield

$$
\begin{aligned}
\mathbb{E} \sup _{t \leq T}\left|N_{\varepsilon}(t)\right| & \lesssim \mathbb{E}\left[N_{\varepsilon}\right](T)^{1 / 2} \leq \mathbb{E} \sup _{s \leq T}\left|y^{(\varepsilon)}(s)\right|\left[\Phi^{(\varepsilon)} \cdot M\right](T)^{1 / 2} \\
& \leq \frac{\alpha}{2} \mathbb{E} \sup _{s \leq T}|y(s)|^{2}+\frac{1}{2 \alpha} \mathbb{E}[\Phi \cdot M](T),
\end{aligned}
$$

for any $\alpha>0$. On the other hand, similarly as above, we have $\mathbb{E}[\Phi \cdot M](T)=\mathbb{E}\langle\Phi \cdot M\rangle(T)=\mathbb{E} \int_{0}^{T}\left|\Phi_{0}(y(s))\right|_{Q_{M}}^{2} d\langle M\rangle(s) \lesssim 1+\int_{0}^{T}\left(\mathbb{E} \sup _{r \leq s}|y(r)|^{2}\right) d s$, which yields

$$
\mathbb{E} \sup _{t \leq T}\left|N_{\varepsilon}(t)\right| \lesssim \frac{\alpha}{2} \mathbb{E} \sup _{s \leq T}|y(s)|^{2}+\frac{1}{\alpha}+\frac{1}{\alpha} \int_{0}^{T}\left(\mathbb{E} \sup _{r \leq s}|y(r)|^{2}\right) d s,
$$

hence also, by (12),

$$
\begin{aligned}
\mathbb{E} \sup _{t \leq T}\left|y^{(\varepsilon)}(t)\right|^{2} \lesssim \mathbb{E} \mid & \left.y_{0}\right|^{2}+\mathbb{E} \sup _{t \leq T} \int_{0}^{t}\left\langle b^{(\varepsilon)}(s), y^{(\varepsilon)}(s)\right\rangle d s \\
& +\frac{1}{\alpha}+\frac{\alpha}{2} \mathbb{E} \sup _{s \leq T}|y(s)|^{2}+\frac{1}{\alpha} \int_{0}^{T}\left(\mathbb{E} \sup _{r \leq s}|y(r)|^{2}\right) d s .
\end{aligned}
$$

Let us now pass to the limit as $\varepsilon$ tends to zero in the previous inequality. We clearly have

$$
\lim _{\varepsilon \rightarrow 0} \mathbb{E} \sup _{t \leq T}\left|y^{(\varepsilon)}(t)\right|^{2}=\mathbb{E} \sup _{t \leq T}|y(t)|^{2}
$$

and, similarly,

$$
\lim _{\varepsilon \rightarrow 0} \mathbb{E} \sup _{t \leq T} \int_{0}^{t}\left\langle b^{(\varepsilon)}(s), y^{(\varepsilon)}(s)\right\rangle d s=\mathbb{E} \sup _{t \leq T} \int_{0}^{t}\langle f(y(s)), y(s)\rangle d s .
$$

In fact, one has $\left\langle b^{(\varepsilon)}(s), y^{(\varepsilon)}(s)\right\rangle \rightarrow\langle f(y(s)), y(s)\rangle \mathbb{P}$-a.s. as $\varepsilon \rightarrow 0$, and

$$
\left|\left\langle b^{(\varepsilon)}(s), y^{(\varepsilon)}(s)\right\rangle\right| \leq|f(y(s))||y(s)| \lesssim 1+|y(s)|^{2},
$$

with $\mathbb{E} \sup _{t \leq T}|y(t)|^{2}<\infty$. We have thus proved

$$
\begin{aligned}
\mathbb{E} \sup _{t \leq T}|y(t)|^{2} \lesssim \mathbb{E}\left|y_{0}\right|^{2}+\frac{1}{\alpha}+\mathbb{E} \sup _{t \leq T} \int_{0}^{t}\langle f(y(s)), y(s)\rangle d s \\
+\frac{\alpha}{2} \mathbb{E} \sup _{t \leq T}|y(t)|^{2}+\frac{1}{\alpha} \int_{0}^{T}\left(\mathbb{E} \sup _{r \leq s}|y(r)|^{2}\right) d s
\end{aligned}
$$

which implies (11) choosing $\alpha$ small enough.

We shall need the following integration-by-parts formula for Hilbert-space-valued semimartingales (cf. [25, §26.9]).

Lemma 8. Let $Z_{1}, Z_{2}$ be two $X$-valued semimartingales. Then one has

$$
\left\langle Z_{1}(t), Z_{2}(t)\right\rangle=\left\langle Z_{1}(0), Z_{2}(0)\right\rangle+\int_{0}^{t}\left\langle Z_{1}(s-), d Z_{2}(s)\right\rangle+\int_{0}^{t}\left\langle Z_{2}(s-), d Z_{1}(s)\right\rangle+\left[Z_{1}, Z_{2}\right](t),
$$


$\mathbb{P}$-a.s. for all $t \geq 0$, where $\left[Z_{1}, Z_{2}\right]$ denotes the quadratic (co)variation of $Z_{1}$ and $Z_{2}$.

Let us also recall, for the reader's convenience, a result of A. Jakubowski on weak convergence of stochastic integrals in infinite dimensions (see [15, Thm. 4]). Given a sequence $\left\{Z^{n}\right\}_{n \in \mathbb{N}}$ of $\mathcal{H}$-valued semimartingales on the corresponding stochastic bases $\left(\Omega, \mathcal{F}, \mathbf{F}^{n}, \mathbb{P}\right)$, one says that $\left\{Z^{n}\right\}_{n \in \mathbb{N}}$ satisfies the UT condition if, for any sequence of $\mathbf{F}^{n}$-adapted $\mathcal{H}$-valued elementary processes $\left\{\zeta^{n}\right\}_{n \in \mathbb{N}}$ uniformly bounded by 1 , the family of random variables

is uniformly tight.

$$
\left\{\int_{0}^{T}\left\langle\zeta^{n}(s-), d Z^{n}(s)\right\rangle\right\}_{n \in \mathbb{N}}
$$

Theorem 9. For each $n \in \mathbb{N}$, let $Z^{n}$ be a $\mathcal{H}$-valued semimartingale with respect to the stochastic basis $\left(\Omega, \mathcal{F}, \mathbf{F}^{n}, \mathbb{P}\right)$ and let $H^{n}$ be $\mathbf{F}^{n}$-adapted and with paths in $D([0, T] \rightarrow$ $\left.\mathcal{L}_{2}(\mathcal{H} \rightarrow X)\right)$. If $\left\{Z^{n}\right\}_{n \in \mathbb{N}}$ satisfies the UT condition and $\left(H^{n}, Z^{n}\right) \rightarrow(H, Z)$ as $n \rightarrow \infty$ in probability in $D\left([0, T] \rightarrow \mathcal{L}_{2}(\mathcal{H} \rightarrow X) \times \mathcal{H}\right)$, then $Z$ is a semimartingale with respect to the natural filtration generated by $(H, Z)$ and

$$
\left(H^{n}, Z^{n}, H_{-}^{n} \cdot Z^{n}\right) \rightarrow\left(H, Z, H_{-} \cdot Z\right)
$$

as $n \rightarrow \infty$ in probability in $D\left([0, T] \rightarrow \mathcal{L}_{2}(\mathcal{H} \rightarrow X) \times \mathcal{H} \times X\right)$.

Note that in [15] the convergences are in law, not in probability. However, everything goes through with convergence in probability as well, just by inspection of the proof (as already observed in a completely analogous setting in [20, p. 1041], cf. also [21, Thm. 5.5]).

Finally, a key role will be played by the following result of Brézis (see [4, Thm. 18]):

Theorem 10. Let $\beta$ be a maximal monotone graph in $\mathbb{R} \times \mathbb{R}$ such that $\operatorname{dom}(\beta)=\mathbb{R}$ and $0 \in \beta(0)$. Let $\left\{f_{n}\right\}_{n \in \mathbb{N}}$ and $\left\{g_{n}\right\}_{n \in \mathbb{N}}$ be sequences of real measurable functions defined on some finite measure space $(\Theta, \mathcal{A}, \mu)$ such that $g_{n} \rightarrow g \mu$-a.e. as $n \rightarrow \infty$, $f_{n}(x) \in \beta\left(g_{n}(x)\right)$ for $\mu$-a.a. $x \in \Theta$, and $f_{n} g_{n} \in L^{1}(\Theta, \mu)$ with $\int_{\Theta} f_{n} g_{n} d \mu<C$ for all $n \in \mathbb{N}$, with $C$ independent of $n$. Then there is a subsequence $\left\{n_{k}\right\}_{k \in \mathbb{N}}$ such that $f_{n_{k}}$ converges to some $f$ in $L^{1}(\Theta, \mu)$ as $k \rightarrow \infty$, and $f(x) \in \beta(g(x))$ for $\mu$-a.a. $x \in \Theta$.

\section{Proof of Theorem 3}

4.1. Some preparations. Before starting to prove the main result, it is helpful to recall a few well-known facts about mild solutions to stochastic wave equations with Lipschitz nonlinearities. In particular, assuming just for the purposes of this subsection that $\beta: \mathbb{R} \rightarrow \mathbb{R}$ is Lipschitz continuous, (3) can be written as the following evolution equation on $H:=H_{0}^{1} \times L^{2}$ :

$$
d U(t)+A U(t) d t+B U(t) d t=G(U(t-)) d M(t), \quad U(0)=\left(u_{0}, v_{0}\right),
$$

where $U(t):=(u(t), v(t))$

$$
\begin{aligned}
A: \operatorname{dom}(A) \subset H & \rightarrow H, \\
(u, v) & \mapsto(-v,-\Delta u),
\end{aligned}
$$


$B: H \ni(u, v) \mapsto(0, \beta(u))$, and $G: H \ni(u, v) \mapsto\left(0, G_{0}(u)\right)$. The operator $-A$ generates a strongly continuous group $S(t)=e^{-t A}$ on $H$, with

$$
S(t)=\left[\begin{array}{cc}
\cos (t \sqrt{-\Delta}) & \frac{1}{\sqrt{-\Delta}} \sin (t \sqrt{-\Delta}) \\
-\sqrt{-\Delta} \sin (t \sqrt{-\Delta}) & \cos (t \sqrt{-\Delta})
\end{array}\right]
$$

(see e.g. [38]), and it is easily seen that (14) admits a unique mild solution, i.e. an adapted càdlàg process $U$ such that the stochastic integral equation

$$
U(t)+\int_{0}^{t} S(t-s) B(U(s)) d s=S(t) U(0)+\int_{0}^{t} S(t-s) G(U(s-)) d M(s),
$$

is satisfied $\mathbb{P}$-a.s. for all $t \in[0, T]$. An elementary computation based on the explicit form of $S(t)$ yields that $U(t)=(u(t), v(t))$ satisfies (15) if and only if it satisfies the alternative form

$$
\left\{\begin{array}{l}
u(t)=u_{0}+\int_{0}^{t} v(s) d s, \\
v(t)+\int_{0}^{t} S_{22}(t-s) \beta(u(s)) d s=S_{21}(t) u_{0}+S_{22}(t) v_{0}+\int_{0}^{t} S_{22}(t-s) G_{0}(u(s-)) d M(s)
\end{array}\right.
$$

$\mathbb{P}$-a.s. for all $t \in[0, T]$, where $S_{i j}(t)$ denotes the $(i, j)$-th entry of the operator matrix $S(t)$. A further computation (which is elementary, apart of having to appeal to a general stochastic Fubini's theorem such as the one in [22]) shows that a mild solution satisfies the Duhamel's formulation

$$
u(t)+\int_{0}^{t} S_{12}(t-s) \beta(u(s)) d s=S_{11}(t) u_{0}+S_{12}(t) v_{0}+\int_{0}^{t} S_{12}(t-s) G_{0}(u(s-)) d M(s) .
$$

As already mentioned, this expression motivates the definition of weak solution in the general case (i.e. without any Lipschitz assumption on $\beta$ ).

4.2. Proof of Theorem 3. Denoting the identity function by $I$, let

$$
\beta_{\lambda}=\frac{1}{\lambda}\left(I-(I+\lambda \beta)^{-1}\right), \quad \lambda>0,
$$

be the Yosida approximation of $\beta$. Recall that the maximal monotonicity of $\beta$ implies that, for any $\lambda>0,(I+\lambda \beta)^{-1}$ is a contraction defined on the whole real line (see e.g. [5, Prop. 2.2]). In particular, $\beta_{\lambda}$ is a function, not a graph. Furthermore, one can prove (see e.g. [5. Prop. 2.6]) that $\beta_{\lambda}: \mathbb{R} \rightarrow \mathbb{R}$ is monotone, Lipschitz continuous with Lipschitz constant bounded above by $2 / \lambda$, and it satisfies $\beta_{\lambda} \in \beta(I+\lambda \beta)^{-1}$.

Consider the regularized equation

$$
d U_{\lambda}(t)+A U_{\lambda}(t) d t+B_{\lambda}\left(U_{\lambda}(t)\right) d t=G\left(U_{\lambda}(t-)\right) d M(t), \quad U_{\lambda}(0)=\left(u_{0}, v_{0}\right),
$$

where $B_{\lambda}:(u, v) \mapsto\left(0, \beta_{\lambda}(u)\right)$ is Lipschitz continous from $H$ to itself. Then (16) admits a unique càdlàg mild solution $U_{\lambda}$ such that

$$
\mathbb{E} \sup _{t \leq T}\left|U_{\lambda}(t)\right|_{H}^{2}<+\infty
$$

(see e.g. [17]). We are now going to establish a priori estimates for $U_{\lambda}=\left(u_{\lambda}, v_{\lambda}\right)$. 
Proposition 11. There exists a positive constant $C$, independent of $\lambda$, such that

$$
\mathbb{E} \sup _{t \leq T}\left(\left|u_{\lambda}(t)\right|_{H_{0}^{1}}^{2}+\left|v_{\lambda}(t)\right|_{L^{2}}^{2}\right)<C .
$$

Proof. By Proposition [7, taking into account that $B_{\lambda}$ is Lipschitz continuous, we have $\mathbb{E} \sup _{t \leq T}\left|U_{\lambda}(t)\right|^{2} \lesssim 1+\mathbb{E}\left|U_{0}\right|^{2}+\mathbb{E} \sup _{t \leq T} \int_{0}^{t}\left\langle-B_{\lambda}\left(U_{\lambda}(s)\right), U_{\lambda}(s)\right\rangle d s+\int_{0}^{T} \mathbb{E} \sup _{r \leq s}\left|U_{\lambda}(r)\right|^{2} d s$, where

$$
\int_{0}^{t}\left\langle B_{\lambda}\left(U_{\lambda}(s)\right), U_{\lambda}(s)\right\rangle d s=\int_{0}^{t}\left\langle\beta_{\lambda}\left(u_{\lambda}(s)\right), v_{\lambda}(s)\right\rangle_{L^{2}} d s .
$$

Let us introduce the Moreau-Yosida approximation of $j$ (recall that $j$ is a positive convex function such that $\beta=\partial j$ ), that is

$$
j_{\lambda}(x):=\inf _{y \in \mathbb{R}}\left(j(y)+\frac{|x-y|^{2}}{2 \lambda}\right), \quad \lambda>0 .
$$

Then one has (see e.g. [1, Thm. 2.2.2]) that $j_{\lambda} \in C^{1}(\mathbb{R}), \beta_{\lambda}=j_{\lambda}^{\prime}$, and $j_{\lambda} \rightarrow j$ pointwise as $\lambda \rightarrow 0$. Moreover, one also has $j_{\lambda} \leq j$ and, obviously, $j_{\lambda} \geq 0$. We can thus write, in view of the identity $u_{\lambda}(t)=u_{0}+\int_{0}^{t} v_{\lambda}(s) d s$, recalling that $j_{\lambda}^{\prime}=\beta_{\lambda}$,

$$
\int_{0}^{t}\left\langle\beta_{\lambda}\left(u_{\lambda}(s)\right), v_{\lambda}(s)\right\rangle_{L^{2}} d s=\int_{D} j_{\lambda}\left(u_{\lambda}(t, x)\right) d x-\int_{D} j_{\lambda}(u(0, x)) d x \geq-\left|j\left(u_{0}\right)\right|_{L^{1}} .
$$

This implies

$$
\mathbb{E} \sup _{t \leq T}\left|U_{\lambda}(t)\right|^{2} \lesssim 1+\mathbb{E}\left|U_{0}\right|^{2}+\mathbb{E}\left|j\left(u_{0}\right)\right|_{L^{1}}+\int_{0}^{T} \mathbb{E} \sup _{r \leq s}\left|U_{\lambda}(r)\right|^{2} d s,
$$

which, by an application of Gronwall's inequality, yields the claim.

Proposition 12. Let $\tilde{u}_{\lambda}(t):=(I+\lambda \beta)^{-1} u_{\lambda}(t), t \in[0, T]$. There exists a positive constant $C$, independent of $\lambda$, such that

$$
\mathbb{E} \int_{0}^{T}\left\langle\beta\left(\tilde{u}_{\lambda}(s)\right), \tilde{u}_{\lambda}(s)\right\rangle_{L^{2}} d s<C .
$$

Proof. We split the proof in three steps.

Step 1. We introduce a regularized version of (16) admitting a strong solution. In particular, setting

$$
F_{\lambda}(t):=\left(0, f_{\lambda}(t)\right):=\left(0, \beta_{\lambda}\left(u_{\lambda}(t)\right)\right), \quad \Gamma_{\lambda}(t):=\left(0, \gamma_{\lambda}(t)\right):=\left(0, G_{0}\left(u_{\lambda}(t-)\right)\right)
$$

for all $t \in[0, T]$, it is clear that $U_{\lambda}$ is the unique mild solution of

$$
d U_{\lambda}(t)+A U_{\lambda}(t) d t+F_{\lambda}(t) d t=\Gamma_{\lambda}(t) d M(t), \quad U_{\lambda}(0)=\left(u_{0}, v_{0}\right),
$$

or equivalently

$$
\left\{\begin{array}{l}
u_{\lambda}(t)=u_{0}+\int_{0}^{t} v_{\lambda}(s) d s \\
v_{\lambda}(t)+\int_{0}^{t} S_{11}(t-s) f_{\lambda}(s) d s=S_{21}(t) u_{0}+S_{11} v_{0}+\int_{0}^{t} S_{11}(t-s) \gamma_{\lambda}(s) d M(s) .
\end{array}\right.
$$


Using the notation $h^{(\varepsilon)}:=(I-\varepsilon \Delta)^{-1} h$ for any "object" $h$ for which the expression makes sense, we may write, recalling that $(I+\varepsilon \Delta)^{-1}$ and $S_{i j}(\cdot), i, j=1,2$, commute,

$$
\left\{\begin{array}{l}
u_{\lambda}^{(\varepsilon)}(t)=u_{0}^{(\varepsilon)}+\int_{0}^{t} v_{\lambda}^{(\varepsilon)}(s) d s \\
v_{\lambda}^{(\varepsilon)}(t)+\int_{0}^{t} S_{11}(t-s) f_{\lambda}^{(\varepsilon)}(s) d s=S_{21}(t) u_{0}^{(\varepsilon)}+S_{11} v_{0}^{(\varepsilon)}+\int_{0}^{t} S_{11}(t-s) \gamma_{\lambda}^{(\varepsilon)}(s) d M(s),
\end{array}\right.
$$

or equivalently, $U_{\lambda}^{(\varepsilon)}(t)=\left(u_{\lambda}^{(\varepsilon)}(t), v_{\lambda}^{(\varepsilon)}(t)\right)$ is the unique mild solution of

$$
d U_{\lambda}^{(\varepsilon)}(t)+A U_{\lambda}^{(\varepsilon)}(t) d t+F_{\lambda}^{(\varepsilon)}(t) d t=\Gamma_{\lambda}^{(\varepsilon)}(t) d M(t), \quad U_{\lambda}^{(\varepsilon)}(0)=\left(u_{0}^{(\varepsilon)}, v_{0}^{(\varepsilon)}\right),
$$

where

$$
F_{\lambda}^{(\varepsilon)}(t)=\left(0, f_{\lambda}^{(\varepsilon)}(t)\right), \quad \Gamma_{\lambda}^{(\varepsilon)}(t)=\left(0, \gamma_{\lambda}^{(\varepsilon)}(t)\right)
$$

for all $t \in[0, T]$. By Lemma 6 we actually have that $U_{\lambda}^{(\varepsilon)}$ is a strong solution of (20).

Step 2. Let $V_{\lambda}^{(\varepsilon)}(t):=\left(0,(I+\lambda \beta)^{-1} u_{\lambda}^{(\varepsilon)}(t)\right), t \in[0, T]$. Then both $U_{\lambda}^{(\varepsilon)}$, as a strong solution of (20), and $V_{\lambda}^{(\varepsilon)}$, are $H$-valued semimartingales, for which the integration-byparts formula (13) yields

$$
\begin{aligned}
\left\langle U_{\lambda}^{(\varepsilon)}(t), V_{\lambda}^{(\varepsilon)}(t)\right\rangle=\langle & \left.U_{\lambda}^{(\varepsilon)}(0), V_{\lambda}^{(\varepsilon)}(0)\right\rangle+\int_{0}^{t}\left\langle U_{\lambda}^{(\varepsilon)}(s-), d V_{\lambda}^{(\varepsilon)}(s)\right\rangle \\
& +\int_{0}^{t}\left\langle V_{\lambda}^{(\varepsilon)}(s-), d U_{\lambda}^{(\varepsilon)}(s)\right\rangle+\left[U_{\lambda}^{(\varepsilon)}, V_{\lambda}^{(\varepsilon)}\right](t) .
\end{aligned}
$$

Taking into account (20) and the definitions of $U_{\lambda}^{(\varepsilon)}, V_{\lambda}^{(\varepsilon)}, F_{\lambda}^{(\varepsilon)}, \Gamma_{\lambda}^{(\varepsilon)}$, we obtain

$$
\begin{aligned}
& \mathbb{E}\left\langle v_{\lambda}^{(\varepsilon)}(t),(I+\lambda \beta)^{-1}\left(u_{\lambda}^{(\varepsilon)}(t)\right)\right\rangle_{L^{2}}=\mathbb{E}\left\langle v_{0}^{(\varepsilon)},(I+\lambda \beta)^{-1} u_{0}^{(\varepsilon)}\right\rangle_{L^{2}} \\
& \quad+\mathbb{E} \int_{0}^{t}\left\langle v_{\lambda}^{(\varepsilon)}(s), d\left((I+\lambda \beta)^{-1}\left(u_{\lambda}^{(\varepsilon)}(s)\right)\right)\right\rangle_{L^{2}} \\
& \quad+\mathbb{E} \int_{0}^{t}\left\langle(I+\lambda \beta)^{-1} u_{\lambda}^{(\varepsilon)}(s), \Delta u_{\lambda}^{(\varepsilon)}(s)\right\rangle_{L^{2}} d s \\
& \quad-\mathbb{E} \int_{0}^{t}\left\langle(I+\lambda \beta)^{-1} u_{\lambda}^{(\varepsilon)}(s),(I-\varepsilon \Delta)^{-1} \beta_{\lambda}\left(u_{\lambda}(s)\right)\right\rangle_{L^{2}} d s \\
& \quad+\mathbb{E} \int_{0}^{t}\left\langle(I+\lambda \beta)^{-1} u_{\lambda}^{(\varepsilon)}(s-), \gamma_{\lambda}^{(\varepsilon)}(s) d M(s)\right\rangle_{L^{2}} d s \\
& +\mathbb{E}\left[v_{\lambda}^{(\varepsilon)},(I+\lambda \beta)^{-1} u_{\lambda}^{(\varepsilon)}\right](t) .
\end{aligned}
$$

Note that, by (19),$\partial_{s} u_{\lambda}^{(\varepsilon)}(s)=v_{\lambda}^{(\varepsilon)}(s)$, and

$$
\begin{aligned}
(I-\lambda \beta)^{-1} u_{\lambda}^{(\varepsilon)}(s) & =u_{\lambda}^{(\varepsilon)}(s)+(I-\lambda \beta)^{-1}\left(u_{\lambda}^{(\varepsilon)}(s)\right)-u_{\lambda}^{(\varepsilon)}(s) \\
& =u_{\lambda}^{(\varepsilon)}(s)-\lambda \beta_{\lambda}\left(u_{\lambda}^{(\varepsilon)}(s)\right),
\end{aligned}
$$


hence

$$
\begin{aligned}
\mathbb{E} \int_{0}^{t} & \left\langle v_{\lambda}^{(\varepsilon)}(s), d\left((I+\lambda \beta)^{-1}\left(u_{\lambda}^{(\varepsilon)}(s)\right)\right)\right\rangle_{L^{2}} d s \\
& =\mathbb{E} \int_{0}^{t}\left\langle v_{\lambda}^{(\varepsilon)}(s), d\left(u_{\lambda}^{(\varepsilon)}(s)-\lambda \beta_{\lambda}\left(u_{\lambda}^{(\varepsilon)}(s)\right)\right)\right\rangle_{L^{2}} \\
& =\mathbb{E} \int_{0}^{t}\left|v_{\lambda}^{(\varepsilon)}(s)\right|_{L^{2}}^{2} d s-\lambda \mathbb{E} \int_{0}^{t}\left\langle v_{\lambda}^{(\varepsilon)}(s), d \beta_{\lambda}\left(u_{\lambda}^{(\varepsilon)}(s)\right)\right\rangle_{L^{2}} .
\end{aligned}
$$

Denoting a family of $C_{c}^{\infty}$ mollifiers by $\left\{\zeta_{\delta}\right\}_{\delta>0}$, and setting $\beta_{\lambda \delta}:=\beta_{\lambda} * \zeta_{\delta}$, one has

and

$$
\mathbb{E} \int_{0}^{t}\left\langle v_{\lambda}^{(\varepsilon)}(s), d \beta_{\lambda \delta}\left(u_{\lambda}^{(\varepsilon)}(s)\right)\right\rangle_{L^{2}} \stackrel{\delta \rightarrow 0}{\longrightarrow} \mathbb{E} \int_{0}^{t}\left\langle v_{\lambda}^{(\varepsilon)}(s), d \beta_{\lambda}\left(u_{\lambda}^{(\varepsilon)}(s)\right)\right\rangle_{L^{2}}
$$

$$
\mathbb{E} \int_{0}^{t}\left\langle v_{\lambda}^{(\varepsilon)}(s), d \beta_{\lambda \delta}\left(u_{\lambda}^{(\varepsilon)}(s)\right)\right\rangle_{L^{2}}=\mathbb{E} \int_{0}^{t}\left\langle v_{\lambda}^{(\varepsilon)}(s), \beta_{\lambda \delta}^{\prime}\left(u_{\lambda}^{(\varepsilon)}(s)\right) v_{\lambda}^{(\varepsilon)}(s)\right\rangle_{L^{2}} d s \geq 0
$$

because, recalling that $\beta_{\lambda}$ is a monotonically increasing function, $\beta_{\lambda \delta}^{\prime}(x)=\beta_{\lambda}^{\prime} * \zeta_{\delta}(x) \geq 0$ for all $x \in \mathbb{R}$. Therefore we have

$$
\mathbb{E} \int_{0}^{t}\left\langle v_{\lambda}^{(\varepsilon)}(s), d\left((I+\lambda \beta)^{-1}\left(u_{\lambda}^{(\varepsilon)}(s)\right)\right)\right\rangle_{L^{2}} d s \leq \mathbb{E} \int_{0}^{t}\left|v_{\lambda}^{(\varepsilon)}(s)\right|_{L^{2}}^{2} d s .
$$

Similarly, we have

$$
\begin{aligned}
\mathbb{E} \int_{0}^{t} & \left\langle(I+\lambda \beta)^{-1}\left(u_{\lambda}^{(\varepsilon)}(s)\right), \Delta u_{\lambda}^{(\varepsilon)}(s)\right\rangle_{L^{2}} d s \\
& =\mathbb{E} \int_{0}^{t}\left\langle u_{\lambda}^{(\varepsilon)}(s), \Delta u_{\lambda}^{(\varepsilon)}(s)\right\rangle_{L^{2}} d s-\mathbb{E} \int_{0}^{t} \lambda\left\langle\beta_{\lambda}\left(u_{\lambda}^{(\varepsilon)}(s)\right), \Delta u_{\lambda}^{(\varepsilon)}(s)\right\rangle_{L^{2}} d s \\
& =-\mathbb{E} \int_{0}^{t}\left\langle\nabla u_{\lambda}^{(\varepsilon)}(s), \nabla u_{\lambda}^{(\varepsilon)}(s)\right\rangle_{L^{2}} d s+\mathbb{E} \int_{0}^{t} \lambda\left\langle\beta_{\lambda}^{\prime}\left(u_{\lambda}^{(\varepsilon)}(s)\right) \nabla u_{\lambda}^{(\varepsilon)}(s), \nabla u_{\lambda}^{(\varepsilon)}(s)\right\rangle_{L^{2}} d s \\
& \leq 2 \mathbb{E} \int_{0}^{t}\left|\nabla u_{\lambda}^{(\varepsilon)}(s)\right|_{L^{2}}^{2} d s,
\end{aligned}
$$

where the term involving $\beta_{\lambda}^{\prime}$ can be interpreted, as above, as limits of more regular expressions obtained replacing $\beta_{\lambda}$ with $\beta_{\lambda \delta}$.

Moreover, by an argument completely similar to one used in the proof of Proposition 7. we get

$$
\mathbb{E} \sup _{t \leq T} \int_{0}^{t}\left\langle(I+\lambda \beta)^{-1} u_{\lambda}^{(\varepsilon)}(s-), \gamma_{\lambda}^{(\varepsilon)}(s) d M(s)\right\rangle_{L^{2}} d s<+\infty,
$$

which implies that the stochastic integral appearing on the right-hand side of (21) is a martingale, hence with expectation zero. Finally, we have that

$$
\left[v_{\lambda}^{(\varepsilon)},(I+\lambda \beta)^{-1} u_{\lambda}^{(\varepsilon)}\right](t)=0
$$

for all $t \in[0, T]$ because, as it follows by (19),

$$
(I+\lambda \beta)^{-1} u_{\lambda}^{(\varepsilon)}(t)=(I+\lambda \beta)^{-1} u_{0}^{(\varepsilon)}+\int_{0}^{t}(I+\lambda \beta)^{-1} v_{\lambda}^{(\varepsilon)}(s) d s
$$


is a process with finite variation and continuous paths. We have thus proved that

$$
\begin{aligned}
\mathbb{E} \int_{0}^{T} & \left\langle(I+\lambda \beta)^{-1} u_{\lambda}^{(\varepsilon)}(s),(I-\varepsilon \Delta)^{-1} \beta_{\lambda}\left(u_{\lambda}(s)\right)\right\rangle_{L^{2}} d s \\
\leq & \mathbb{E}\left\langle v_{0}^{(\varepsilon)},(I+\lambda \beta)^{-1} u_{0}^{(\varepsilon)}\right\rangle_{L^{2}}+\mathbb{E}\left|\left\langle v_{\lambda}^{(\varepsilon)}(T),(I+\lambda \beta)^{-1} u_{\lambda}^{(\varepsilon)}(T)\right\rangle_{L^{2}}\right| \\
& +\mathbb{E} \int_{0}^{T}\left|v_{\lambda}^{(\varepsilon)}(s)\right|_{L^{2}}^{2} d s+2 \mathbb{E} \int_{0}^{T}\left|\nabla u_{\lambda}^{(\varepsilon)}(s)\right|_{L^{2}}^{2} d s .
\end{aligned}
$$

Furthermore, Cauchy-Schwarz' inequality and the contractivity of $(I+\lambda \beta)^{-1}$ and $(I-$ $\varepsilon \Delta)^{-1}$ yield

$$
\begin{aligned}
& \mathbb{E}\left\langle v_{0}^{(\varepsilon)},(I+\lambda \beta)^{-1} u_{0}^{(\varepsilon)}\right\rangle_{L^{2}} \leq\left(\mathbb{E}\left|v_{0}\right|_{L^{2}}^{2}\right)^{1 / 2}\left(\mathbb{E}\left|u_{0}\right|_{L^{2}}^{2}\right)^{1 / 2}, \\
& \mathbb{E}\left|\left\langle v_{\lambda}^{(\varepsilon)}(T),(I+\lambda \beta)^{-1} u_{\lambda}^{(\varepsilon)}(T)\right\rangle_{L^{2}}\right| \leq\left(\mathbb{E}\left|v_{\lambda}(T)\right|_{L^{2}}^{2}\right)^{1 / 2}\left(\mathbb{E}\left|u_{\lambda}(T)\right|_{L^{2}}^{2}\right)^{1 / 2} \\
& \leq\left(\mathbb{E} \sup _{t \leq T}\left|v_{\lambda}(t)\right|_{L^{2}}^{2}\right)^{1 / 2}\left(\mathbb{E} \sup _{t \leq T}\left|u_{\lambda}(t)\right|_{L^{2}}^{2}\right)^{1 / 2}, \\
& \mathbb{E} \int_{0}^{T}\left|v_{\lambda}^{(\varepsilon)}(s)\right|_{L^{2}}^{2} d s \leq \mathbb{E} \int_{0}^{T}\left|v_{\lambda}(s)\right|_{L^{2}}^{2} d s \leq T \mathbb{E} \sup _{t \leq T}\left|v_{\lambda}(t)\right|_{L^{2}}^{2}, \\
& \mathbb{E} \int_{0}^{T}\left|\nabla u_{\lambda}^{(\varepsilon)}(s)\right|_{L^{2}} d s \leq \mathbb{E} \int_{0}^{T}\left|\nabla u_{\lambda}(s)\right|_{L^{2}} d s \leq T \mathbb{E} \sup _{t \leq T}\left|u_{\lambda}(t)\right|_{H_{0}^{1}}^{2} .
\end{aligned}
$$

Appealing to Proposition 11, we infer that there exists a constant $C$, independent of $\varepsilon$ and of $\lambda$, such that

$$
\mathbb{E} \int_{0}^{t}\left\langle(I+\lambda \beta)^{-1} u_{\lambda}^{(\varepsilon)}(s),(I-\varepsilon \Delta)^{-1} \beta_{\lambda}\left(u_{\lambda}(s)\right)\right\rangle_{L^{2}} d s<C .
$$

Step 3. We shall now pass to the limit as $\varepsilon \rightarrow 0$ in the last inequality. Since the operator $(I+\lambda \beta)^{-1}$ is bounded, we have that

$$
\lim _{\varepsilon \rightarrow 0}\left\langle(I+\lambda \beta)^{-1} u_{\lambda}^{(\varepsilon)}(s),(I-\varepsilon \Delta)^{-1} \beta_{\lambda}\left(u_{\lambda}(s)\right)\right\rangle_{L^{2}}=\left\langle(I+\lambda \beta)^{-1} u_{\lambda}(s), \beta_{\lambda}\left(u_{\lambda}(s)\right)\right\rangle_{L^{2}}
$$

$\mathbb{P}$-a.s. for all $s \in[0, T]$. On the other hand, the contractivity of $(I+\lambda \beta)^{-1}$ and $(I-\varepsilon \Delta)^{-1}$, the Lipschitz continuity of $\beta_{\lambda}$, and Cauchy-Schwarz' inequality yield

$$
\begin{gathered}
\mathbb{E} \int_{0}^{T}\left|\left\langle(I+\lambda \beta)^{-1} u_{\lambda}^{(\varepsilon)}(s),(I-\varepsilon \Delta)^{-1} \beta_{\lambda}\left(u_{\lambda}(s)\right)\right\rangle_{L^{2}}\right| d s \\
\lesssim \mathbb{E} \int_{0}^{T}\left(1+\left|u_{\lambda}(s)\right|_{L^{2}}^{2}\right) d s<\infty .
\end{gathered}
$$

Hence, by the dominated convergence theorem, we obtain

$$
\begin{aligned}
\mathbb{E} \int_{0}^{T}\langle(I & \left.+\lambda \beta)^{-1} u_{\lambda}(s), \beta_{\lambda}\left(u_{\lambda}(s)\right)\right\rangle_{L^{2}} d s \\
& =\lim _{\varepsilon \rightarrow 0} \mathbb{E} \int_{0}^{T}\left\langle(I+\lambda \beta)^{-1} u_{\lambda}^{(\varepsilon)}(s),(I-\varepsilon \Delta)^{-1} \beta_{\lambda}\left(u_{\lambda}(s)\right)\right\rangle_{L^{2}} d s<C,
\end{aligned}
$$


where $C$ is the same constant (independent of $\lambda$ ) appearing in (22), thus completing the proof, upon recalling that $\beta_{\lambda} \in \beta(I+\lambda \beta)^{-1}$.

Proof of Theorem 3. Let $\left(u_{\lambda}, v_{\lambda}\right)$ be the solution of the regularized equation (16), for which one has

$$
u_{\lambda}(t)=u_{0}+\int_{0}^{t} v_{\lambda}(s) d s
$$

for all $t \in[0, T]$, and, by Proposition 11,

$$
\mathbb{E} \sup _{t \leq T}\left|v_{\lambda}(t)\right|_{L^{2}}^{2}<C
$$

for a constant $C$ that does not depend on $\lambda$. This implies, for any $\eta>0$,

$$
\mathbb{P}\left(\sup _{t \leq T}\left|v_{\lambda}(t)\right|_{L^{2}}^{2}>\eta\right) \leq \frac{1}{\eta} \mathbb{E}\left(\sup _{t \leq T}\left|v_{\lambda}(t)\right|_{L^{2}}^{2}\right)<\frac{C}{\eta},
$$

thus also

$$
\lim _{\eta \rightarrow \infty} \sup _{\lambda} \mathbb{P}\left(\sup _{t \leq T}\left|v_{\lambda}(t)\right|_{L^{2}}^{2}>\eta\right)=0 .
$$

Let us now show that, for any $t \in[0, T]$ and $\delta>0$, there exists a compact subset $K=K(t, \delta)$ of $L^{2}$ such that $\mathbb{P}\left(u_{\lambda}(t) \in K\right)>1-\delta$. In fact, denoting by $B_{R}$ the ball of radius $R$ centered at the origin of $H_{0}^{1}$ and recalling that $H_{0}^{1}$ is compactly embedded in $L^{2}$, we have that $B_{R}$ is a compact subset of $L^{2}$, and

$$
\mathbb{P}\left(u_{\lambda}(t) \in B_{R}\right)=1-\mathbb{P}\left(\left|u_{\lambda}(t)\right|_{H_{0}^{1}}>R\right) \geq 1-\frac{1}{R^{2}} \mathbb{E} \sup _{t \leq T}\left|u_{\lambda}(t)\right|_{H_{0}^{1}}^{2}>1-\frac{C}{R^{2}},
$$

i.e. it is enough to choose $R=\sqrt{C / \delta}$ and $K=B_{R}$. This observation and (24) imply, thanks to a corollary to a theorem of Rebolledo (see e.g. [26, §II.4.4]), that $\left\{u_{\lambda}\right\}_{\lambda}$ is uniformly tight in $D\left([0, T] \rightarrow L^{2}\right)$. By Skorohod's representation theorem (see e.g. $[3, \S 8.5])$, there exists a probability space $(\bar{\Omega}, \overline{\mathcal{F}}, \overline{\mathbb{P}})$ and a sequence of random vectors $\xi_{n}:=\left(\bar{u}_{n}, \bar{M}_{n}\right) \in D\left([0, T] \rightarrow L^{2} \times L^{2}\right)$ such that $\xi_{n} \rightarrow \xi:=(\bar{u}, \bar{M}) \overline{\mathbb{P}}$-a.s. as $n \rightarrow \infty$, and the laws of $\xi_{n}$ and $\left(u_{\lambda_{n}}, M\right)$ coincide for each $n$, for some subsequence $\left\{\lambda_{n}\right\}_{n \in \mathbb{N}}$ of $\lambda$. Let us set

$$
\mathcal{B}_{n}=\left(\bar{\Omega}, \overline{\mathcal{F}}, \overline{\mathbf{F}}_{n}, \overline{\mathbb{P}}\right), \quad \mathcal{B}=(\bar{\Omega}, \overline{\mathcal{F}}, \overline{\mathbf{F}}, \overline{\mathbb{P}}),
$$

where $\overline{\mathbf{F}}_{n}$ and $\overline{\mathbf{F}}$ are the filtrations generated by $\left(\bar{u}_{n}, \bar{M}_{n}\right)$ and $(\bar{u}, \bar{M})$, respectively. Then $\bar{u}_{n}$ is $\overline{\mathbf{F}}_{n}$-adapted and càdlàg, and $\bar{u}$ is $\overline{\mathbf{F}}$-adapted and càdlàg, since $\bar{u}_{n}$ converges $\overline{\mathbb{P}}$-a.s. to $\bar{u}$ as $n \rightarrow \infty$ in the Skorohod topology.

Let us assume, for the time being, that the process $\bar{M}_{n}$ is a $\overline{\mathbf{F}}_{n}$-martingale for each $n$. Then the process $\bar{M}$ is a $\overline{\mathbf{F}}$-martingale by a slight modification of the proof of [14, Prop. IX.1.10], taking into account [op. cit., Prop. IX.1.12 and Rmk. VI.1.10], as well as the obvious inequality

$$
\sup _{t \leq T} \overline{\mathbb{E}}\left|\bar{M}_{n}(t)\right|^{2}=\sup _{t \leq T} \mathbb{E}|M(t)|^{2}<\infty
$$

(where $\overline{\mathbb{E}}$ denotes expectation with respect to $\overline{\mathbb{P}}$ on the stochastic basis $\mathcal{B}_{n}$ ), which implies that $\bar{M}_{n}$ is uniformly integrable. On the other hand, a completely similar argument proves that $\bar{M}_{n}$ is indeed a $\mathbf{F}_{n}$-martingale for each $n$ : fix $n$ and set $M^{k}:=M$ and $Y^{k}:=u_{n}, k \in \mathbb{N}$, so that $\left(Y^{k}, M^{k}\right)$ trivially converges in law to $\left(\bar{u}_{n}, \bar{M}_{n}\right)$, which has the 
same law of $\left(u_{n}, M\right)$. Since $\left\{M^{k}\right\}_{k \in \mathbb{N}}$ is obviously uniformly integrable, we conclude that $\bar{M}_{n}$ is a martingale with respect to the filtration generated by $\left(\bar{u}_{n}, \bar{M}_{n}\right)$, as it follows by the above mentioned results of [14.

Setting $\beta_{n}:=\beta_{\lambda_{n}}$, we also have that, for each fixed $n, \bar{u}_{n}$ solves $\overline{\mathbb{P}}$-a.s. the integral equation

$$
\begin{aligned}
& \bar{u}_{n}(t)+\int_{0}^{t} \frac{1}{\sqrt{-\Delta}} \sin ((t-s) \sqrt{-\Delta}) \beta_{n}\left(\bar{u}_{n}(s)\right) d s \\
= & \cos (t \sqrt{-\Delta}) u_{0}+\frac{1}{\sqrt{-\Delta}} \sin (t \sqrt{-\Delta}) v_{0}+\int_{0}^{t} \frac{1}{\sqrt{-\Delta}} \sin ((t-s) \sqrt{-\Delta}) G_{0}\left(\bar{u}_{n}(s-)\right) d \bar{M}_{n}(s),
\end{aligned}
$$

because the same equation is satisfied with $\bar{u}_{n}$ and $\bar{M}_{n}$ replaced by $u_{\lambda_{n}}$ and $M$, respectively (for instance by an argument such as the one used in [18, p. 89]).

Lemma 13. There is a subsequence $\left\{n_{k}\right\}_{k \in \mathbb{N}}$ such that $\bar{u}_{n_{k}} \rightarrow \bar{u} \overline{\mathbb{P}} \otimes$ Leb-a.e. on $\bar{\Omega} \times[0, T] \times D$.

Proof. Note that we clearly have

$$
\overline{\mathbb{E}} \int_{0}^{T}\left|\bar{u}_{n}(t)-\bar{u}(t)\right|_{L^{1}} d t \lesssim \overline{\mathbb{E}} \int_{0}^{T}\left|\bar{u}_{n}(t)-\bar{u}(t)\right|_{L^{2}} d t .
$$

Since (23) implies $\bar{u}_{n} \in C\left([0, T] \rightarrow L^{2}\right)$, upon recalling that the Skorokhod topology on $D\left([0, T] \rightarrow L^{2}\right)$ induces the uniform topology on its subspace $C\left([0, T] \rightarrow L^{2}\right)$, we have that $\bar{u}_{n}$ converges to $\bar{u} \overline{\mathbb{P}}$-a.s. in $C\left([0, T] \rightarrow L^{2}\right)$. That is

$$
\sup _{t \leq T}\left|\bar{u}_{n}(t)-\bar{u}(t)\right|_{L^{2}} \rightarrow 0
$$

$\overline{\mathbb{P}}$-a.s. as $n \rightarrow \infty$. Set $X_{n}:=\int_{0}^{T}\left|\bar{u}_{n}(t)-\bar{u}(t)\right|_{L^{2}} d t$. By (27), the sequence $X_{n}$ converges to 0 in probability. Assume for the moment that

$$
\sup _{n} \overline{\mathbb{E}}\left|X_{n}\right|_{L^{2}}^{2}<+\infty
$$

Then $\left\{X_{n}\right\}_{n \in \mathbb{N}}$ is uniformly integrable and $\overline{\mathbb{E}} X_{n} \rightarrow 0$ as $n \rightarrow \infty$, whence, by (26), $\lim _{n \rightarrow \infty} \bar{u}_{n}=\bar{u}$ in $L^{1}(\bar{\Omega} \times D \times[0, T])$, thus also $\bar{u}_{n_{k}} \rightarrow \bar{u} \overline{\mathbb{P}} \otimes$ Leb-a.e. on $\bar{\Omega} \times D \times[0, T]$ as $k \rightarrow \infty$, along a subsequence $\left\{n_{k}\right\}_{k \in \mathbb{N}}$.

It remains to justify (28). Observe that

$$
\begin{aligned}
\overline{\mathbb{E}}\left|X_{n}\right|^{2} & \lesssim \overline{\mathbb{E}} \int_{0}^{T}\left|\bar{u}_{n}(t)-\bar{u}(t)\right|_{L^{2}}^{2} d t \\
& \lesssim \overline{\mathbb{E}} \int_{0}^{T}\left|\bar{u}_{n}(t)\right|_{L^{2}}^{2}+\overline{\mathbb{E}} \int_{0}^{T}|\bar{u}(t)|_{L^{2}}^{2} d t \\
& =: I_{1}+I_{2},
\end{aligned}
$$

and

$$
I_{1} \lesssim \sup _{n} \sup _{t \leq T} \overline{\mathbb{E}}\left|\bar{u}_{n}(t)\right|_{L^{2}}^{2}<+\infty
$$


by Proposition 11 and the fact that $\bar{u}_{n}$ has the same law as $u_{\lambda_{n}}$. On the other hand, (27) implies

$$
\int_{0}^{T}\left|\bar{u}_{n}(t)\right|_{L^{2}}^{2} d t \rightarrow \int_{0}^{T}|\bar{u}(t)|_{L^{2}}^{2} d t
$$

$\overline{\mathbb{P}}$-a.s. as $n \rightarrow \infty$, hence, by Fatou's lemma,

$$
I_{2}=\overline{\mathbb{E}} \int_{0}^{T}|\bar{u}(t)|_{L^{2}}^{2} d t \leq \liminf _{n} \overline{\mathbb{E}} \int_{0}^{T}\left|\bar{u}_{n}(t)\right|_{L^{2}}^{2} d t \lesssim \sup _{n} \sup _{t \leq T} \overline{\mathbb{E}}\left|\bar{u}_{n}(t)\right|_{L^{2}}^{2}<+\infty,
$$

which concludes the proof.

Let us now consider the convergence of the stochastic integrals in (25).

Lemma 14. One has, for each $t \in[0, T]$,

$$
\int_{0}^{t} S_{12}(t-s) G_{0}\left(\bar{u}_{n}(s-)\right) d \bar{M}_{n}(s) \rightarrow \int_{0}^{t} S_{12}(t-s) G_{0}(\bar{u}(s-)) d \bar{M}(s)
$$

in probability as $n \rightarrow \infty$.

Proof. By a basic trigonometric identity we can write

$$
\sin ((t-s) \sqrt{-\Delta})=\sin (t \sqrt{-\Delta}) \cos (-s \sqrt{-\Delta})+\cos (t \sqrt{-\Delta}) \sin (-s \sqrt{-\Delta}) .
$$

Therefore, setting

$$
H_{n}^{1}(s):=\cos (-s \sqrt{-\Delta}) G_{0}\left(\bar{u}_{n}(s)\right), \quad H_{n}^{2}(s):=\sin (-s \sqrt{-\Delta}) G_{0}\left(\bar{u}_{n}(s)\right),
$$

for all $s \in[0, T]$, we get

$$
\begin{aligned}
\int_{0}^{t} S_{12}(t-s) G_{0}\left(\bar{u}_{n}(s-)\right) d \bar{M}_{n}(s)= & S_{12}(t) \int_{0}^{t} H_{n}^{1}(s-) d \bar{M}_{n}(s) \\
& +\frac{1}{\sqrt{-\Delta}} \cos (t \sqrt{-\Delta}) \int_{0}^{t} H_{n}^{2}(s-) d \bar{M}_{n}(s) .
\end{aligned}
$$

By the continuity of $G_{0}$ and the boundedness of $\cos (-s \sqrt{-\Delta})$ and $\sin (-s \sqrt{-\Delta})$, we infer $\left(H_{n}^{1}, \bar{M}_{n}\right) \rightarrow\left(H^{1}, \bar{M}\right)$ and $\left(H_{n}^{2}, \bar{M}_{n}\right) \rightarrow\left(H^{2}, \bar{M}\right)$ in $D\left([0, T] \rightarrow \mathcal{L}_{2}^{Q} \times L^{2}\right)$ in probability as $n \rightarrow \infty$, where

$$
H^{1}(s):=\cos (-s \sqrt{-\Delta}) G_{0}(\bar{u}(s)), \quad H^{2}(s):=\sin (-s \sqrt{-\Delta}) G_{0}(\bar{u}(s))
$$

for all $s \in[0, T]$. Let us now show that the sequence $\left\{\bar{M}_{n}\right\}_{n \in \mathbb{N}}$ satisfies the UT condition specified just before the statement of Theorem 9. In fact, denoting a sequence of elementary processes as in 93 , mutatis mutandis, by $\left\{\zeta_{n}\right\}_{n \in \mathbb{N}}$, (2) and Markov's inequality yield

$$
\overline{\mathbb{P}}\left(\left|\int_{0}^{t}\left\langle\zeta_{n}(s-), d \bar{M}_{n}(s)\right\rangle_{\mathcal{H}}\right|>\eta\right) \leq T \frac{1}{\eta^{2}} \overline{\mathbb{E}} \sup _{s \leq t}\left|\zeta_{n}(s)\right|_{\mathcal{H}}^{2} \leq \frac{T}{\eta^{2}},
$$

which implies, observing that the upper bound just obtained does not depend on $n$,

$$
\lim _{\eta \rightarrow \infty} \sup _{n} \overline{\mathbb{P}}\left(\left|\int_{0}^{t}\left\langle\zeta_{n}(s-), d \bar{M}_{n}(s)\right\rangle_{\mathcal{H}}\right|>\eta\right)=0
$$


for all $t \leq T$, i.e. the UT condition is verified. At this point we can apply Jakubowski's result Theorem 9 to deduce that

$$
\begin{aligned}
\int_{0} H_{1}^{n}(s-) d \bar{M}_{n}(s) & \rightarrow \int_{0} \cos (-s \sqrt{-\Delta}) G_{0}(\bar{u}(s-)) d \bar{M}(s), \\
\int_{0} H_{2}^{n}(s-) d \bar{M}_{n}(s) & \rightarrow \int_{0} \sin (-s \sqrt{-\Delta}) G_{0}(\bar{u}(s-)) d \bar{M}(s),
\end{aligned}
$$

in probability in $D\left([0, T] \rightarrow L^{2}\right)$ as $n \rightarrow \infty$, which allows to conclude, in view of (29).

We can now conclude the proof of Theorem 3. By Proposition 12 and Skorohod's representation, there exists a constant $C$, independent of $n$, such that

$$
\overline{\mathbb{E}} \int_{0}^{T}\left\langle\beta\left(J_{n} \bar{u}_{n}\right), J_{n} \bar{u}_{n}\right\rangle_{L^{2}} d s<C,
$$

where $J_{n}:=\left(I+\lambda_{n} \beta\right)^{-1}$. Therefore, by Theorem 10, we have that there exists $g \in \beta(\bar{u})$ such that, on a further subsequence, still denoted by $n$,

$$
\beta_{n}\left(\bar{u}_{n}\right) \rightarrow g \quad \text { in } L^{1}(\bar{\Omega} \times[0, T] \times D, \overline{\mathbb{P}} \otimes \text { Leb })
$$

as $n \rightarrow \infty$. In particular, passing to a further subsequence if necessary,

$$
\beta_{n}\left(\bar{u}_{n}\right) \rightarrow g \quad \text { in } L^{1}\left([0, T] \rightarrow L^{1}\right)
$$

$\overline{\mathbb{P}}_{\text {-a.s. as }} n \rightarrow \infty$. Let us define the scale of Hilbert spaces

$$
\mathfrak{H}^{m}:=\mathfrak{H}_{1}^{m} \times \mathfrak{H}_{2}^{m}:=\operatorname{dom}\left((I-\Delta)^{m / 2}\right) \times \operatorname{dom}\left((I-\Delta)^{(m-1) / 2}\right), \quad m \in \mathbb{R},
$$

where, for each $m \in \mathbb{R}$, dom $\left((I-\Delta)^{m / 2}\right)$ is endowed with the norm

$$
\|x\|_{m}=\left|(I-\Delta)^{m / 2} x\right|_{L^{2}} .
$$

For instance, $\mathfrak{H}^{0}=L^{2} \times H^{-1}$ and $\mathfrak{H}^{1}=H_{0}^{1} \times L^{2}$, which are the traditional Hilbert spaces on which the strongly continuous group $\{S(t)\}_{t \in \mathbb{R}}$ associated to the linear wave equation is considered. Since, for each $s \in \mathbb{R},(I-\Delta)^{s / 2}: \mathfrak{H}^{m} \rightarrow \mathfrak{H}^{m-s}$ is an isometric isomorphism (considered componentwise) and $(I-\Delta)^{s / 2}$ commutes with $S(t)$ for any $s$, $t$, one immediately verifies that $\{S(t)\}_{t \in \mathbb{R}}$ can be extended (or restricted) to a strongly continuous group on $\mathfrak{H}^{m}$ for all $m \in \mathbb{R}$ (cf. [12, §II.5] for a related general scheme to extend semigroups of operators to so-called Sobolev towers). By classical Sobolev embedding theorems (see e.g. [24]), there exists $m>0$ such that $L^{1} \subset \operatorname{dom}((I-$ $\Delta)^{-(m+1) / 2}$ ) with continuous embedding, therefore, in view of (30),

$$
\beta_{n}\left(\bar{u}_{n}\right) \rightarrow g \quad \text { in } L^{1}\left([0, T] \rightarrow \mathfrak{H}_{2}^{-m}\right)
$$

$\overline{\mathbb{P}}$-a.s. as $n \rightarrow \infty$, and so

$$
\int_{0}^{t} S_{12}(t-s) \beta_{n}\left(\bar{u}_{n}(s)\right) d s \rightarrow \int_{0}^{t} S_{12}(t-s) g(s) d s
$$

$\overline{\mathbb{P}}$-a.s. as $n \rightarrow \infty$ by continuity of $\left\{S_{12}(t)\right\}_{t \in \mathbb{R}}$ in $\mathfrak{H}_{2}^{-m}$. 
Summing up, we have obtained that $(\bar{u}, g)$ solves $\overline{\mathbb{P}}$-a.s. the equation

$$
\begin{aligned}
& \bar{u}(t)+\int_{0}^{t} \frac{1}{\sqrt{-\Delta}} \sin ((t-s) \sqrt{-\Delta}) g(s) d s \\
& =\cos (t \sqrt{-\Delta}) u_{0}+\frac{1}{\sqrt{-\Delta}} \sin (t \sqrt{-\Delta}) v_{0}+\int_{0}^{t} \frac{1}{\sqrt{-\Delta}} \sin ((t-s) \sqrt{-\Delta}) G_{0}(\bar{u}(s-)) d \bar{M}(s),
\end{aligned}
$$

where all random vectors have to be considered as taking values in $\mathfrak{H}_{2}^{-m}$, thus proving that $\bar{u}$ is a (probabilistically) weak solution of (1), in the sense of Definition 1 .

\section{REFERENCES}

1. V. Barbu, Analysis and control of nonlinear infinite-dimensional systems, Academic Press Inc., Boston, MA, 1993. MR MR1195128 (93j:49002)

2. V. Barbu, G. Da Prato, and M. Röckner, Existence of strong solutions for stochastic porous media equation under general monotonicity conditions, Ann. Probab. 37 (2009), no. 2, 428-452. MR MR2510012

3. V. I. Bogachev, Measure theory. Vol. II, Springer-Verlag, Berlin, 2007. MR MR2267655 (2008g:28002)

4. H. Brézis, Monotonicity methods in Hilbert spaces and some applications to nonlinear partial differential equations, Contributions to nonlinear functional analysis (Proc. Sympos., Math. Res. Center, Univ. Wisconsin, Madison, Wis., 1971), Academic Press, New York, 1971, pp. 101-156. MR MR0394323 (52 \#15126)

5. __ Opérateurs maximaux monotones et semi-groupes de contractions dans les espaces de Hilbert, North-Holland Publishing Co., Amsterdam, 1973. MR MR0348562 (50 \#1060)

6. R. Carmona and D. Nualart, Random nonlinear wave equations: smoothness of the solutions, Probab. Theory Related Fields 79 (1988), no. 4, 469-508. MR 90f:60112

7. P.-L. Chow, Stochastic wave equations with polynomial nonlinearity, Ann. Appl. Probab. 12 (2002), no. 1, 361-381. MR MR1890069 (2003e:60135)

8. D. Conus and R.C. Dalang, The non-linear stochastic wave equation in high dimensions, Electron. J. Probab. 13 (2008), no. 22, 629-670. MR 2399293 (2009c:60170)

9. R. C. Dalang, The stochastic wave equation, A minicourse on stochastic partial differential equations, Lecture Notes in Math., vol. 1962, Springer, Berlin, 2009, pp. 39-71. MR 2508773 (2010h:60191)

10. R.C. Dalang and N.E. Frangos, The stochastic wave equation in two spatial dimensions, Ann. Probab. 26 (1998), no. 1, 187-212. MR 99c:60127

11. R.C. Dalang and C. Mueller, Some non-linear S.P.D.E.'s that are second order in time, Electron. J. Probab. 8 (2003), no. 1, 21 pp. (electronic). MR MR1961163 (2004a:60118)

12. K.-J. Engel and R. Nagel, One-parameter semigroups for linear evolution equations, Graduate Texts in Mathematics, vol. 194, Springer-Verlag, New York, 2000. MR MR1721989 (2000i:47075)

13. J. Jacod, Calcul stochastique et problèmes de martingales, Lecture Notes in Mathematics, vol. 714, Springer, Berlin, 1979. MR MR542115 (81e:60053)

14. J. Jacod and A. N. Shiryaev, Limit theorems for stochastic processes, second ed., Springer-Verlag, Berlin, 2003. MR MR1943877 (2003j:60001)

15. A. Jakubowski, Continuity of the Ito stochastic integral in Hilbert spaces, Stochastics Stochastics Rep. 59 (1996), no. 3-4, 169-182. MR MR1427737 (98a:60036)

16. A. Karczewska and J. Zabczyk, A note on stochastic wave equations, Evolution equations and their applications in physical and life sciences (Bad Herrenalb, 1998), Lecture Notes in Pure and Appl. Math., vol. 215, Dekker, New York, 2001, pp. 501-511. MR 1818028 (2002b:60112)

17. P. Kotelenez, A stopped Doob inequality for stochastic convolution integrals and stochastic evolution equations, Stochastic Anal. Appl. 2 (1984), no. 3, 245-265. MR MR757338 (86k:60096)

18. N. V. Krylov, Controlled diffusion processes, Springer-Verlag, New York, 1980. MR 82a:60062 
19. N. V. Krylov and B. L. Rozovskiı̌, Stochastic evolution equations, Current problems in mathematics, Vol. 14 (Russian), Akad. Nauk SSSR, Vsesoyuz. Inst. Nauchn. i Tekhn. Informatsii, Moscow, 1979, pp. 71-147, 256. MR MR570795 (81m:60116)

20. Th. G. Kurtz and Ph. Protter, Weak limit theorems for stochastic integrals and stochastic differential equations, Ann. Probab. 19 (1991), no. 3, 1035-1070. MR MR1112406 (92k:60130)

21. __ Weak convergence of stochastic integrals and differential equations. II. Infinite-dimensional case, Probabilistic models for nonlinear partial differential equations (Montecatini Terme, 1995), Lecture Notes in Math., vol. 1627, Springer, Berlin, 1996, pp. 197-285. MR MR1431303 (98h:60074)

22. J. A. León, Stochastic Fubini theorem for semimartingales in Hilbert space, Canad. J. Math. 42 (1990), no. 5, 890-901. MR MR1081001 (92f:60096)

23. J.-P. Lepeltier and B. Marchal, Problème des martingales et équations différentielles stochastiques associées à un opérateur intégro-différentiel, Ann. Inst. H. Poincaré Sect. B (N.S.) 12 (1976), no. 1, 43-103. MR MR0413288 (54 \#1403)

24. V. G. Maz'ja, Sobolev spaces, Springer-Verlag, Berlin, 1985. MR MR817985 (87g:46056)

25. M. Métivier, Semimartingales, Walter de Gruyter \& Co., Berlin, 1982. MR MR688144 (84i:60002)

26. S Stochastic partial differential equations in infinite-dimensional spaces, Scuola Normale Superiore, Pisa, 1988. MR MR982268 (90a:60112)

27. A. Millet and P.-L. Morien, On a nonlinear stochastic wave equation in the plane: existence and uniqueness of the solution, Ann. Appl. Probab. 11 (2001), no. 3, 922-951. MR MR1865028 (2002i:60118)

28. A. Millet and M. Sanz-Solé, A stochastic wave equation in two space dimension: smoothness of the law, Ann. Probab. 27 (1999), no. 2, 803-844. MR 2001e:60130

29. M. Ondreját, Existence of global mild and strong solutions to stochastic hyperbolic evolution equations driven by a spatially homogeneous Wiener process, J. Evol. Equ. 4 (2004), no. 2, 169-191. MR MR2059301 (2005e:60134)

30. _ Existence of global martingale solutions to stochastic hyperbolic equations driven by a spatially homogeneous Wiener process, Stoch. Dyn. 6 (2006), no. 1, 23-52. MR MR2210680 (2006m:60081)

31. E. Pardoux, Equations aux derivées partielles stochastiques nonlinéaires monotones, Ph.D. thesis, Université Paris XI, 1975.

32. J. Pellaumail, Solutions faibles et semi-martingales, Seminar on Probability, XV (Univ. Strasbourg, Strasbourg, 1979/1980) (French), Lecture Notes in Math., vol. 850, Springer, Berlin, 1981, pp. 561586. MR MR622588 (83c:60087)

33. Sz. Peszat, The Cauchy problem for a nonlinear stochastic wave equation in any dimension, J. Evol. Equ. 2 (2002), no. 3, 383-394. MR 2003k:60157

34. Sz. Peszat and J. Zabczyk, Stochastic evolution equations with a spatially homogeneous Wiener process, Stochastic Process. Appl. 72 (1997), no. 2, 187-204. MR MR1486552 (99k:60166)

35. _ Nonlinear stochastic wave and heat equations, Probab. Theory Related Fields 116 (2000), no. 3, 421-443. MR 2001f:60071

36. Stochastic partial differential equations with Lévy noise, Cambridge University Press, Cambridge, 2007. MR MR2356959

37. C. Prévôt and M. Röckner, A concise course on stochastic partial differential equations, Lecture Notes in Mathematics, vol. 1905, Springer, Berlin, 2007. MR MR2329435

38. I. E. Segal, The global Cauchy problem for a relativistic scalar field with power interaction, Bull. Soc. Math. France 91 (1963), 129-135. MR MR0153967 (27 \#3928)

39. J. Shatah and M. Struwe, Geometric wave equations, Courant Lecture Notes in Mathematics, vol. 2, New York University Courant Institute of Mathematical Sciences, New York, 1998. MR MR1674843 (2000i:35135)

40. A. V. Skorokhod, Studies in the theory of random processes, Izdat. Kiev. Univ., Kiev, 1961, in Russian. English Translation: Addison-Wesley, 1965. MR MR0185619 (32 \#3082a)

41. J. B. Walsh, An introduction to stochastic partial differential equations, École d'été de probabilités de Saint-Flour, XIV—1984, Lecture Notes in Math., vol. 1180, Springer, Berlin, 1986, pp. 265-439. 
(C. Marinelli) Facoltà di Economia, Università di Bolzano, I-39100 Bolzano, Italy And Dipartimento di Matematica, Università di Trento, I-38123 Trento, Italy.

$U R L:$ http://www . uni-bonn.de/ $\operatorname{cm} 788$

(L. Quer-Sardanyons) Departament de Matemàtiques, Universitat Autònoma de Barcelona, 08193 Cerdanyola del Vallès (Barcelona), Catalunya, Spain.

$U R L:$ http://www . mat. uab.cat/ quer 